\title{
TiN-coating formation by pulsed Nd:YAG laser irradiation of titanium in nitrogen
}

\author{
Daniel Höche, Hendrik Schikora, Hayo Zutz, \\ Andreas Emmel, Robert Queitsch, Peter Schaaf
}

(C) The Author(s) 2008

\begin{abstract}
Titanium was treated by pulsed Nd:YAG laser irradiation in nitrogen atmosphere, which led to nitrogen in-diffusion and TiN coating formation. The thickness of the TiN films was about $1.2 \mu \mathrm{m}$ and the coatings had a universal hardness of about $11 \mathrm{GPa}$. The layers were investigated by X-ray diffraction at grazing incidence and resonant nuclear reaction analysis for nitrogen depth profiling. Fitting of the experimental depth profiles gave information about the physical processes (diffusion time and depth) with respect to the achieved hardness. The microscopic properties like lattice constants and the variation of the nitrogen content were evaluated. A relationship between laser scan parameters and coating properties could be revealed. Thus, it was possible to determine the physical limits such as film thickness, nitrogen content, and hardness of this direct laser synthesis.
\end{abstract}

Keywords Titanium, Hardness, Melting depth, Laser synthesis, Titanium nitride, Coatings

\section{Introduction}

Nitriding and carburizing of metals is a known technique to improve hardness and tribological properties of metals. ${ }^{1}$ Plasma and gas nitriding, for example, are

D. Höche $(\bowtie)$, H. Schikora, H. Zutz, P. Schaaf

II. Physikalisches Institut, Universität Göttingen,

Friedrich-Hund-Platz 1, 37077 Gottingen, Germany

e-mail: dhoeche@gwdg.de; dhoeche@uni-goettingen.de

A. Emmel

FH Amberg-Weiden, Lasertechnik, Kaiser-Wilhelm-Ring

23, 92224 Amberg, Germany

R. Queitsch

ARGES GmbH, Industrieplanung und Lasertechnik,

Kirchplatz 4, 92507 Nabburg, Germany established methods of metal surface treatments. ${ }^{2}$ Alternatively, it is possible to treat the surface by laser irradiation in reactive atmospheres and to synthesize hard coatings on metals directly. ${ }^{3-5}$ Previous works showed the successful synthesis of titanium nitride coatings. ${ }^{6-8}$ Extensive investigations were done by Mori ${ }^{9}$ and György ${ }^{10,11}$ by means of pulsed Nd:YAG laser irradiation. The advantage of this method is that the processing time is reduced down to some seconds per $\mathrm{cm}^{2}$ sample area. However, surface roughness and inhomogeneity remain the main problems. Thus, a set of laser treatment parameters has to be found in order to optimize the coating properties. Nitriding of titanium has also been tried by Xue, ${ }^{12,13}$ who carried out similar investigations with a different setup.

By the laser treatments presented here, $\mathrm{TiN}_{x}$ coatings have been formed, too. These coatings were analyzed by X-ray diffraction (XRD), nitrogen depth profiling by resonant nuclear reaction analysis (RNRA), and hardness measurements. The correlations between the scan parameters and the resulting coating properties will be pointed out next.

\section{Experiments}

\section{Titanium preparation}

Commercial $\alpha$-titanium (purity $>99.98 \%$ ) was used for the treatments. The $1-\mathrm{mm}$-thick sheets were cut into pieces of $15 \times 15 \mathrm{~mm}^{2}$ and used without any further treatment in the as-received state.

\section{Sample treatment}

The titanium samples were placed in a chamber, which was first evacuated and then filled with pure nitrogen (99.999\% purity) at a pressure of 3 bar. Laser irradi- 


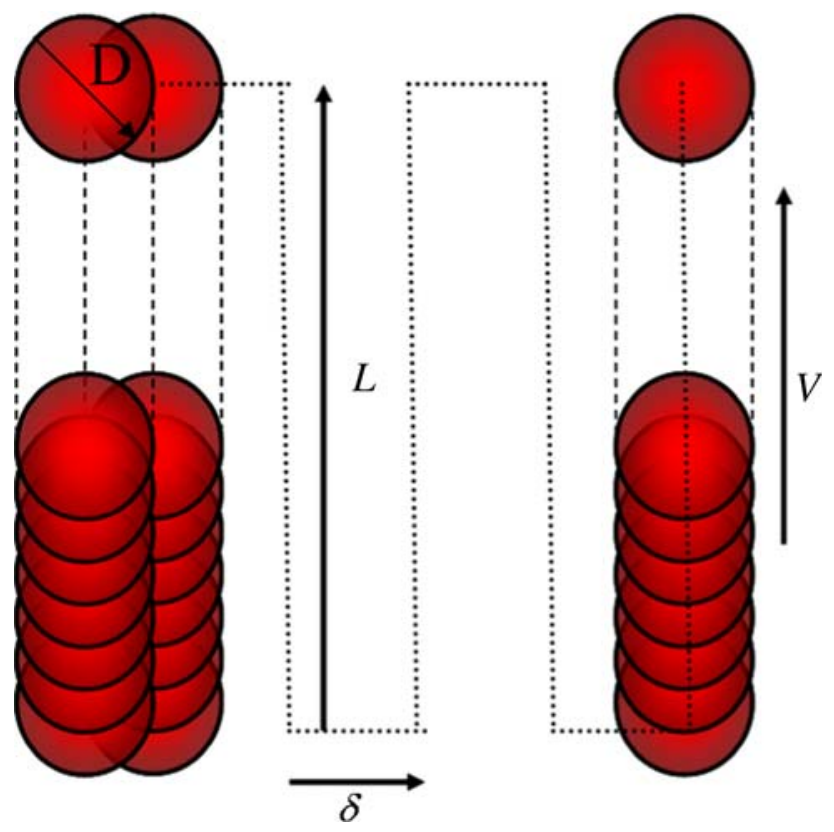

Fig. 1: Meandering laser treatment of the titanium samples. The treatment parameters are the lateral shift $\delta$ between subsequent line scans, the scan velocity $v$, and spot diameter $D$. The lines had a total travel length $L$ of $10 \mathrm{~mm}$

ations were carried out in Amberg using the first harmonic of a Q-switched Nd:YAG laser $(\lambda=532 \mathrm{~nm})$. The pulses of $6 \mathrm{~ns}$ duration (FWHM) and $100 \mathrm{~Hz}$ repetition rate had a mean pulse energy of about $40 \mathrm{~mJ}$. As indicated in Fig. 1, an optical scanner moved the laser beam across the samples in a meandering fashion.

Combining the pulse repetition rate $f_{\text {pulse }}$ and the geometric parameters of the meandering, a dimensionless parameter for a quantitative description of the treatment of each surface element can be derived.

$\Sigma=n \frac{D^{2} \cdot f_{\text {pulse }}}{v \cdot \delta}$

Equation (1) defines this dimensionless overlap parameter $\Sigma$ for the parametric description of the process, where $D$ gives the spot diameter at the sample surface, $n$ the number of scans, $v$ is the scan velocity, and $\delta$ the lateral shift after each line scan of $10 \mathrm{~mm}$ length. In principle, $\Sigma$ represents the total number of laser pulses hitting each surface element of the treated sample. This parameter does not properly take into account the temporal and spatial structure of the treatment, e.g. the different elapsed time between subsequent pulses and pulses of the next line scan.

The scan speed $v$ was varied between 10 and $50 \mathrm{~mm} / \mathrm{s}^{-1}$. Moreover, the energy density active at the sample surface was changed by varying the spot size $D$ from 0.7 to $1.15 \mathrm{~mm}$. The lateral shift $\delta$ between two subsequent scans was varied from 0.05 to $0.2 \mathrm{~mm}$.

Due to the laser irradiation, a plasma formed on top of the liquid titanium surface resulting in the dissociation of

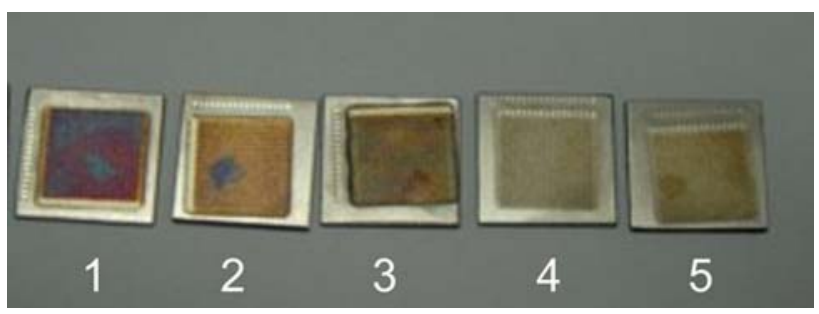

Fig. 2: Photographs of laser nitrided titanium. The treatment parameters are given in Table 1

Table 1: Scan parameters of selected samples

\begin{tabular}{cccc} 
Sample & $n$ & $\delta(\mathrm{mm})$ & $\Sigma$ \\
\hline 1 & 2 & 0.10 & 66.7 \\
2 & 1 & 0.10 & 33.3 \\
3 & 1 & 0.05 & 66.7 \\
4 & 1 & 0.20 & 16.7 \\
5 & 1 & 0.15 & 22.3 \\
\hline
\end{tabular}

All scans were done with a pulse frequency of $f=100 \mathrm{~Hz}$, a spot diameter $D=1 \mathrm{~mm}$, and a scan velocity of $v=30 \mathrm{~mm} / \mathrm{s}$ in pure nitrogen gas at a pressure of 3 bar. The number of repeated (identical) treatments (scans) $n$, lateral shift $\delta$, and overlap parameter $\Sigma$ are given

nitrogen. Consequently, nitrogen diffused into the melt and after solidification a TiN coating had developed. Examples of the produced samples are shown in Fig. 2, which clearly shows that the treatment parameters given in Table 1 strongly influence the treatment results.

\section{Analysis}

The microstructure of the layers was analyzed by XRD in grazing incidence geometry (GIXRD), using a Bruker AXS diffractometer equipped with a $\mathrm{Cu}-\mathrm{K}_{\alpha}$ tube and a thin film attachment. Peak analyses yielded lattice constants, nitrogen contents, stress, and crystallite size.

Nitrogen depth profiling was carried out by means of the resonant nuclear reaction analysis (RNRA) employing the reaction ${ }^{15} \mathrm{~N}(\mathrm{p}, \alpha \gamma){ }^{12} \mathrm{C} \cdot{ }^{14}$ The measurements were performed at the Göttingen IONAS accelerator. ${ }^{15}$ Details are given in reference (7). The nitrogen depth profiles were analysed assuming diffusion profiles.

The microhardness depth profiles were measured with a nanoindenter (Fischerscope HV100). It operates with a Vickers diamond tip and a maximum force of $1 \mathrm{~N}^{7}$ Scanning electron microscopy (SEM) was performed for surface analyses (FEI Nova 600). Transmission electron microscopy (TEM) was employed (Philips CM200-FEG) for selected samples in order to gain information about film thickness, microstructure, morphology, and homogeneity. A focussed ion beam setup was used for the sample preparation. 


\section{Results}

\section{$X$-ray diffractometry}

A typical XRD diffraction spectrum is shown in Fig. 3 and exhibits cubic titanium nitride ${ }^{16}$ in addition to the virgin $\alpha$-Ti. Comparable results for continuous $\mathrm{Nd}$ :YAG laser-treated titanium are reported in the literature. ${ }^{9}$ For the $3^{\circ}$ grazing incidence geometry that was used here, the information depth is about half a micron. By comparing the peak areas, a TiN content of about $70 \%$ in the surface is revealed for sample 1 .

Using this type of diffraction geometry, the lattice parameter $a$ can be determined. For this purpose, the peak positions have to be fitted to the experimental data and the stresses in the coatings have to be taken into account. The macroscopic stress and the lattice constant can be calculated from the lattice plane distances $d^{17}$ by using equations (2) and (3).

$\frac{\Delta d}{d_{0}}=\frac{\sigma}{E}\left[(1+v) \sin ^{2} \Psi-2 v\right] \quad$ with $\quad \Psi=\Theta-\omega$

$\Delta d$ is the variation of the lattice plane distance from the unstressed distance $d_{0}, \sigma$ is the stress, $\omega$ is the tilting angle, $E$ describes the Young modulus $(280 \mathrm{GPa}$ for $\left.\operatorname{TiN}_{0.67}\right),{ }^{18} v$ is the Poisson ratio $(v=0.25)^{19}$ and thus $\Psi$ is the angle between the surface normal and the scattering vector. Together with Bragg's law, equation (2) results in equation (3) for a cubic crystal:

$$
\begin{aligned}
\sin \Theta_{0} & =\sin \left(\Theta+\frac{\sigma}{E} \tan \Theta\left[(1+v) \sin ^{2} \Psi-2 v\right]\right) \\
& =\frac{\lambda}{2} \sqrt{\frac{h^{2}+k^{2}+l^{2}}{d_{0}^{2}}}
\end{aligned}
$$

The experimental $(h k l)$ data from the five TiN reflections were linearly fitted to equation (3) and

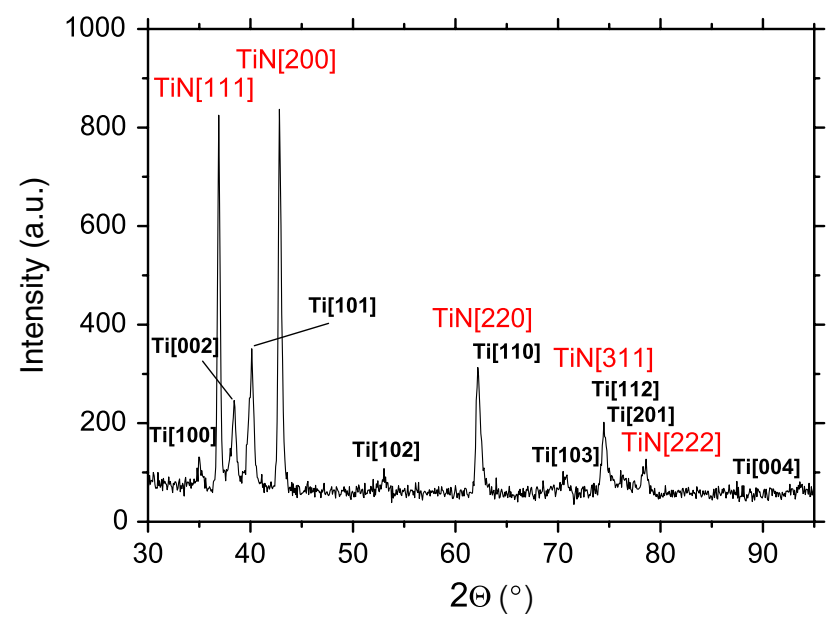

Fig. 3: XRD spectrum at $3^{\circ}$ grazing incidence for sample 1 . The peak areas give about $70 \%$ TiN and $30 \% \alpha-\mathrm{Ti}$ resulted in a macroscopic stress $\sigma=-1.1 \mathrm{GPa}$ and a lattice constant $a=4.224 \AA$. Additionally, information about the nitrogen content can be obtained by means of Vegard's law ${ }^{20}$ as shown in equation (4), which is based on experimental data: ${ }^{21}$

$a=(4.1925+x \cdot 0.0467) \AA$.

The $\mathrm{N}$ content amounts to $x=0.67$. Lee et al. ${ }^{18}$ measured a value of $4.2256 \AA$ for $\mathrm{TiN}_{0.67}$ on a $\mathrm{MgO}$ substrate, which is in good agreement with our results. Crystallite size and microstrain are resolved elsewhere. $^{22}$

\section{Nitrogen depth profiles}

Diffusion-like nitrogen contents are shown in Figs. 4 and 5. They were measured by the RNRA method, which has a maximum detection depth of around $500 \mathrm{~nm}$ due to the limited proton energy of the used accelerator. Thus, not the whole modified layer is seen by this method. Very important is the fact that nearly 50 at.\% nitrogen concentrations are observed close to the surface. The same effect was observed in a previous study by György. ${ }^{10}$ A mathematical description of these profiles can be given by a superposition of two complementary error function profiles (solutions of diffusion equation). The boundary conditions were assumed to be constant with three concentrations, $c_{0}$, $c_{l}$, and $c_{s}$. This solution as given in equation (5) contains two diffusion lengths, a long-range (index $l$ ) and a short-range (index $s$ ) diffusion. The long range diffusion is mainly describing the macroscopic nitrogen diffusion and determines the film thickness and the macroscopic properties. In contrast, the short range diffusion describes more the near surface effects as

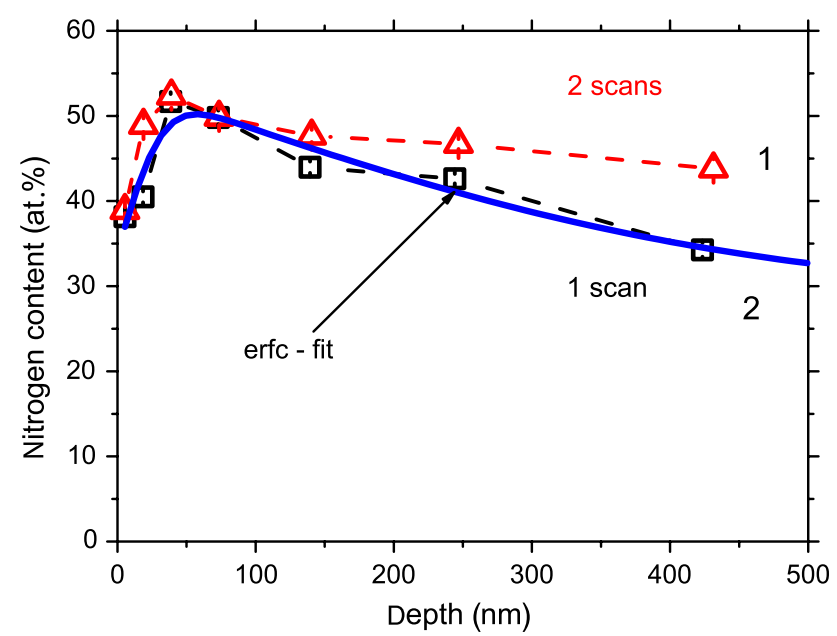

Fig. 4: Nitrogen depth profiles for one and two scans $(D=$ $1 \mathrm{~mm}, \delta=0.1 \mathrm{~mm}, v=30 \mathrm{~mm} / \mathrm{s}$, and $f=100 \mathrm{~Hz}$ ) and an example for a complementary error function fit (solid line) 


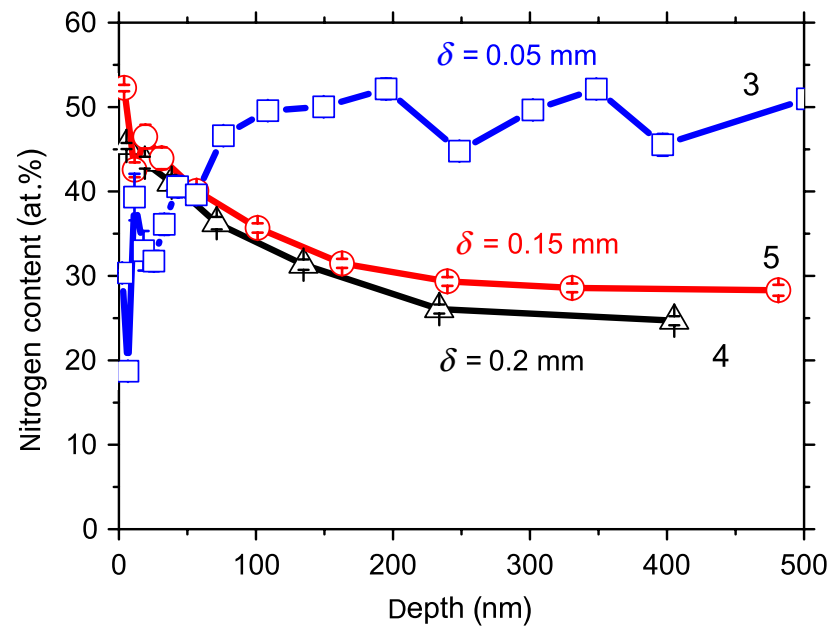

Fig. 5: Nitrogen depth profiles for different shifts $(D=$ $1 \mathrm{~mm}, v=30 \mathrm{~mm} / \mathrm{s}$, and $f=100 \mathrm{~Hz}$ )

nitrogen adsorption and incorporation or even oxidation effects.

$$
\begin{aligned}
c= & c_{0}+c_{1}\left[1-\operatorname{erf}\left(\frac{x}{\sqrt{4 D t_{l}}}\right)\right] \\
& +\left(c_{s}-c_{0}\right)\left[1-\operatorname{erf}\left(\frac{x}{\sqrt{4 D t_{s}}}\right)\right]
\end{aligned}
$$

In our case, the parameters determining the characteristics of the layer are the diffusion lengths $L_{l, s}$. Using the relation $L_{l, s}=\sqrt{4 D t_{l, s}}$ it is possible to obtain information about the diffusion time.

The mean diffusion coefficient was assumed to be $2 \times 10^{-4} \mathrm{~cm}^{2} / \mathrm{s}$. This value correlates with the work of Shuja et al. ${ }^{23}$ in a temperature range near the boiling point. Thus, the value is just averaged over the whole process. Some conclusions can be drawn from these diffusion ranges. For thin layers it is about three times slower than for others. Additionally, the diffusion rate should slow down in greater depths as the temperatures should also be lower there.

The results from fitting the five examples are summarized in Table 2, which shows that the diffusion time is three orders of magnitude higher than the pulse duration. The short range values are $L_{s}=30 \mathrm{~nm}$ and $t_{s}$ $=17 \mathrm{~ns}$, and they do not change significantly with the varying parameters.

They correlate with the time for which titanium stays liquid. Furthermore, it can be confirmed that no significant improvement is achieved by repeating the treatment, even though the nitrogen content is slightly higher in deeper layers. Only remelting and a weak mixing effect occurs. The surface quality cannot be improved, which is proved by the hardness measurements. Moreover, there is a correlation between the overlap parameter and the diffusion length. Figure 5 shows the influence that changing the lateral shift has.
Table 2: Diffusion data of selected samples (errors are about $10 \%$ )

\begin{tabular}{cccc}
$\begin{array}{c}\text { Sample } \\
\Sigma\end{array}$ & $\begin{array}{c}\text { Overlap } \\
(\mu \mathrm{m})\end{array}$ & $\begin{array}{c}\text { Diffusion length } L_{l} \\
(\mu \mathrm{s})\end{array}$ \\
\hline 1 & 66.7 & 0.53 & 3.51 \\
2 & 33.3 & 0.50 & 3.13 \\
3 & 66.7 & 0.79 & 7.80 \\
4 & 16.7 & 0.17 & 0.36 \\
5 & 22.3 & 0.19 & 0.45 \\
\hline
\end{tabular}

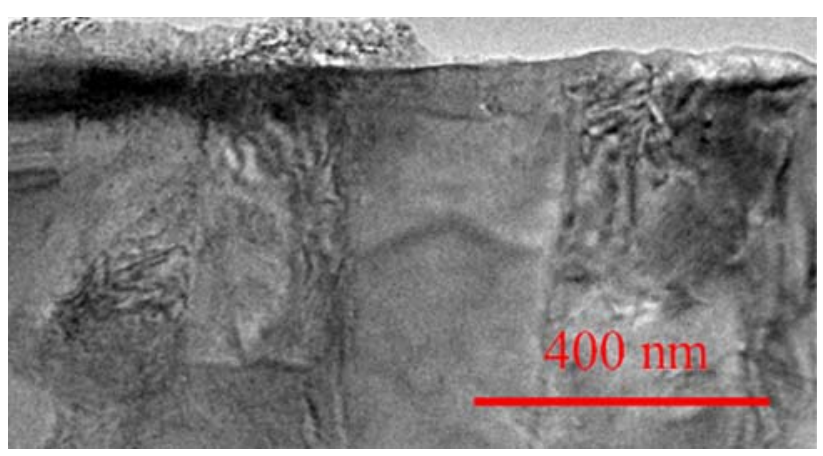

Fig. 6: TEM cross-section micrograph of sample 2

Smaller values resulted in a strong increase in film thickness but also in processing time.

\section{Microscopy}

In order to get more information about the microstructure of the coating, SEM and TEM were used to create surface and cross-section micrographs. Figure 6 shows a cross section of sample 2. The layer is determined by the heat flux and the convection of the melt. A large grain of about $200 \mathrm{~nm}$ is observed. The growth direction of this crystallite is perpendicular to the surface. Figure 7 shows the surface; its roughness is found to be determined by the melt dynamics. There are some droplets which should be avoided, in order to improve the layer properties. On the other hand, no cracks were found, despite the fact that the laser energy was very high.

\section{Hardness}

Hardness depth profiles of the treated samples were measured by the nanoindentation technique, which give information about the film (coating) hardness and the sample hardness. The measured hardness depth profiles are presented in Fig. 8. All the depth profiles exhibit a steep increase of the hardness within the first few hundred nanometers from the surface. Then, they exhibit a maximum of the hardness before slowly decreasing to the pure titanium hardness value. 
The effect of low hardness close to the very surface is a result of the high surface roughness and known for nanoindentation measurements of rough samples. Oxidation effects might also influence the hardness profile at the very surface, but such will be neglected

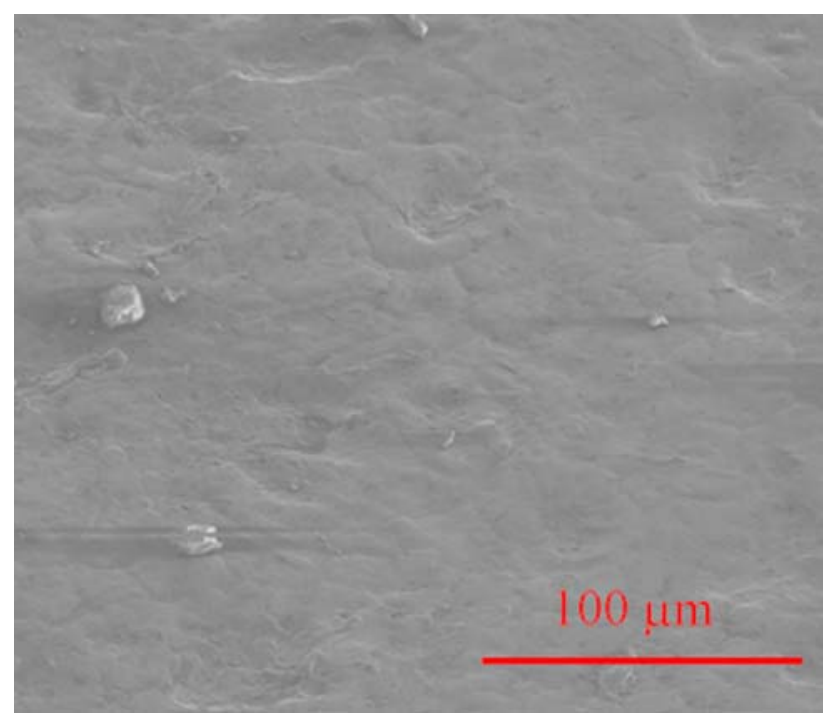

Fig. 7: SEM surface micrograph of sample 2
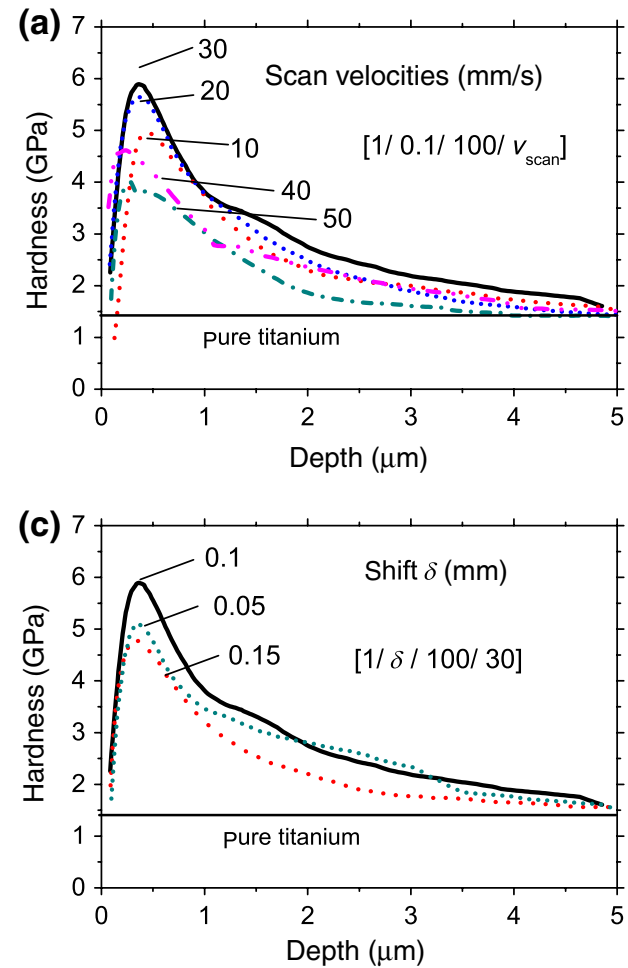

here, as they should have a small influence and a small penetration depth. ${ }^{24}$

The maximum values of the measured hardness profiles $H_{C-\max }$ are summarized in Table 3 . For all the samples, the maximum hardness is in the same range, which slightly exceeds $6 \mathrm{GPa}$. This seems to be a limit for the process.

Jönsson and Hogmark ${ }^{25}$ developed a model to describe the hardness for a system of a hard thin film on a soft substrate, which is expressed by:

$H_{C}=H_{S}+\left[2 k \frac{t}{d}-\left(k \frac{t}{d}\right)^{2}\right]\left(H_{F}-H_{S}\right)$

The measured hardness $H_{C}$ is a function of the substrate hardness $H_{S}$, the film thickness $t$, the indentation depth $d$, the film hardness $H_{F}$, and the constant $k$. In the present case (Fischerscope HV100 with a Vickers diamond), $k$ should have a value between 0.07 and 0.14. According to the geometry of the used indenter, $k$ was set to $0.14 .^{25}$ The coating thickness was determined by means of cross-section micrographs and comparisons with the fits. Due to the inhomogeneous coating properties, average values were used.

The model of Jönsson and Hogmark ${ }^{25}$ is valid only for $d>t,{ }^{26}$ i.e. only for the region right of the coating thickness $t$. Thus, the fits presented here are performed only for indention depths higher than $t$. An example for
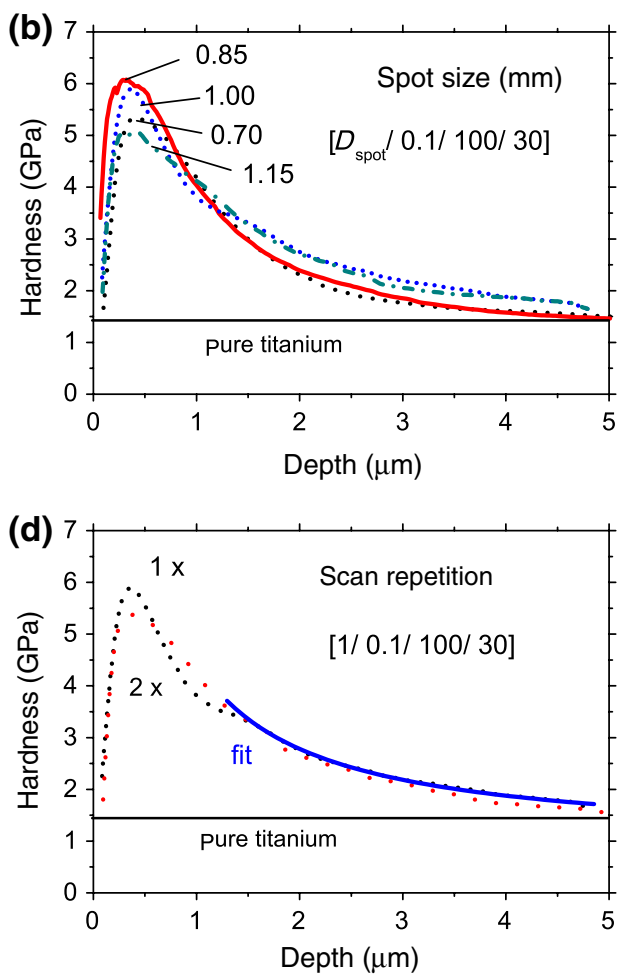

Fig. 8: Influence of (a) the scan velocity $v_{\text {scan, }}$ (b) the spot size $D_{\text {spot, }}$ (c) the lateral shift $\delta$, and (d) the scan repetition $n$ on the measured hardness. In (d), an example of a Jönsson and Hogmark fit is shown (fit). For all plots, the parameters are given by the notation $D_{\text {spot }}(\mathrm{mm}) / \delta(\mathrm{mm}) / f(\mathrm{~Hz}) / v_{\text {scan }}(\mathrm{mm} / \mathrm{s})$ 
Table 3: Maximum values of the measured hardness data $\boldsymbol{H}_{C \text {-max }}$ of the investigated samples and their overlap parameter $\Sigma$

\begin{tabular}{|c|c|c|c|c|c|}
\hline Parameters & $\Sigma$ & Changed parameter & $H_{C-\max }(\mathrm{GPa})$ & $H_{F}(\mathrm{GPa})$ & $t(\mu \mathrm{m})$ \\
\hline \multirow[t]{5}{*}[1.00/0.10/100/v]{} & 100 & $10 \mathrm{~mm} / \mathrm{s}$ & 4.98 & $8.05 \pm 0.1$ & $1.4 \pm 0.1$ \\
\hline & 50 & $20 \mathrm{~mm} / \mathrm{s}$ & 5.66 & $10.98 \pm 0.1$ & $1.3 \pm 0.1$ \\
\hline & 33.3 & $30 \mathrm{~mm} / \mathrm{s}$ & 5.87 & $11.82 \pm 0.1$ & $1.2 \pm 0.1$ \\
\hline & 25 & $40 \mathrm{~mm} / \mathrm{s}$ & 4.62 & $9.78 \pm 0.1$ & $1.0 \pm 0.1$ \\
\hline & 20 & $50 \mathrm{~mm} / \mathrm{s}$ & 3.93 & $8.92 \pm 0.1$ & $0.8 \pm 0.1$ \\
\hline \multirow[t]{4}{*}[D_{\text{spot}}/0.10/100/30]{} & 16.3 & $0.70 \mathrm{~mm}$ & 5.35 & $9.33 \pm 0.1$ & $1.4 \pm 0.1$ \\
\hline & 24.1 & $0.85 \mathrm{~mm}$ & 6.08 & $10.51 \pm 0.1$ & $1.3 \pm 0.1$ \\
\hline & 33.3 & $1.00 \mathrm{~mm}$ & 5.87 & $11.82 \pm 0.1$ & $1.2 \pm 0.1$ \\
\hline & 44.1 & $1.15 \mathrm{~mm}$ & 5.09 & $9.64 \pm 0.1$ & $1.2 \pm 0.1$ \\
\hline \multirow[t]{3}{*}[1.00/\delta/100/30]{} & 66.7 & $0.05 \mathrm{~mm}$ & 5.08 & $8.77 \pm 0.1$ & $1.3 \pm 0.1$ \\
\hline & 33.3 & $0.10 \mathrm{~mm}$ & 5.87 & $11.82 \pm 0.1$ & $1.2 \pm 0.1$ \\
\hline & 22.3 & $0.15 \mathrm{~mm}$ & 4.78 & $7.93 \pm 0.1$ & $1.2 \pm 0.1$ \\
\hline \multirow[t]{2}{*}{ No. scans $[1.00 / 0.10 / 100 / 30]$} & 33.3 & 1 & 5.87 & $11.82 \pm 0.1$ & $1.2 \pm 0.1$ \\
\hline & 66.7 & 2 & 5.35 & $9.56 \pm 0.1$ & $1.7 \pm 0.1$ \\
\hline
\end{tabular}

The scan parameters are shown as $D_{\text {spot }}(\mathrm{mm}) / \delta(\mathrm{mm}) / f(\mathrm{~Hz}) / v_{\text {scan }}(\mathrm{mm} / \mathrm{s})$. The film thickness $t$ was estimated from cross sections and the films hardness $H_{F}$ were determined by fitting the measured hardness curves with equation (6)
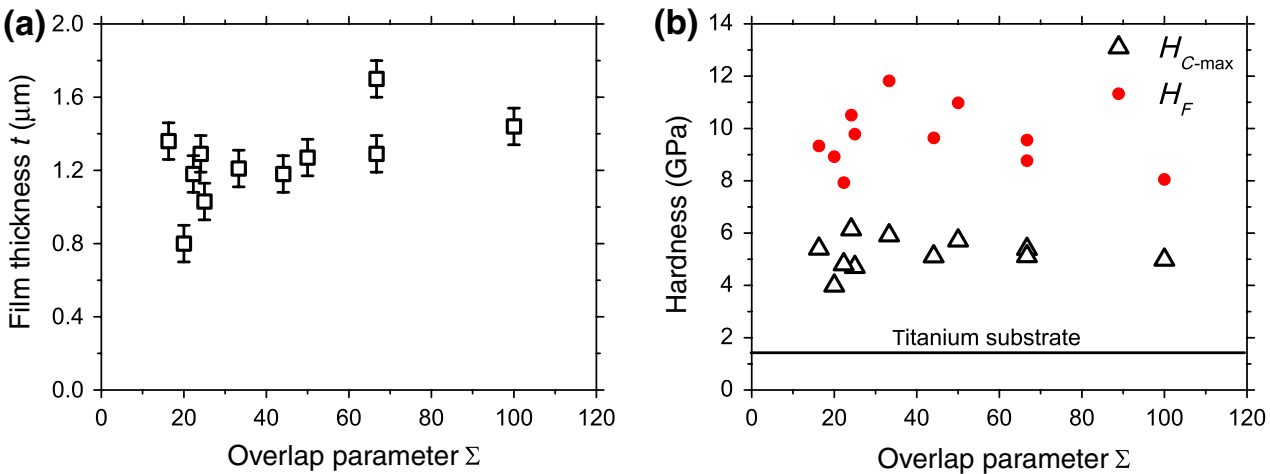

Fig. 9: Dependence of the film thickness (a) and the film hardness (b) on the overlap parameter. The film thickness seems to be limited by the melting depth $(\approx 2 \mu \mathrm{m})$. The maximum film hardness is found around $\Sigma=30$, where the balance between induced cracks and melting is optimal

such a fit is given in Fig. 8d (fit). Table 3 summarizes all the results from the fits.

First, the scan velocity was varied and a maximum of hardness can be observed at $30 \mathrm{~mm} / \mathrm{s}$ (Fig. 8a). These results depend on the balance between melting time and convection influences. For high velocities the radiation time was too short for deep melting, thus the film thickness decreases. But for slow scans, there were remelting and convection effects, which induced cracks. Changes in the spot size are simply related to changes in the energy density per area. For all spot sizes the intensity is above the evaporation threshold, thus there are almost the same conditions for the melt. The reason for this is the high energy of the laser beam. In this case, there are only small changes in the hardness.

The influence of the lateral shift is similar to that of the velocity. A maximum hardness can be observed at a lateral shift of $0.1 \mathrm{~mm}$. The arguments are the same as for the velocity. Figure $8 \mathrm{~d}$ shows the hardness profiles for different scans and an example of a fit according to equation (6). The same effect could be observed when the scans were repeated. This is practically unnecessary, because the nitrogen diffusion and uptake is principally saturated. Only the nitrogen profile in deeper layers has changed as a result of reheating and mixing.

Figure 9 shows the dependence of the film thickness and hardness on the overlap parameter, as resulting from the hardness curve fits and correspond to the shown assumptions. In the used model, the film hardness $H_{F}$ is always very similar to the maximum hardness $H_{C-\max }$, as the measured maximum always appears close to $d=k t$, a depth of approximately $0.5 \mu \mathrm{m}$. The film thickness and the nitrogen depth could not be correlated, because of the limited 
measurement range of the RNRA method. The film thickness is below $2 \mu \mathrm{m}$. At an overlap value of 30 the best layer properties were synthesized.

\section{Conclusions}

Nd:YAG laser treatment of titanium in a reactive nitrogen atmosphere resulted in thin $\delta$ - $\mathrm{TiN}_{x}$ coatings of about 1-2 $\mu \mathrm{m}$ thickness. Depending on the scan parameters, an overall hardness of nearly $6 \mathrm{GPa}$ could be reached, which corresponds to a film hardness of about $11 \mathrm{GPa}$. Additional investigations of nitrogen depth profiles were performed, which supply information about the diffusion process and the physical relationships. A diffusion time of some $\mu$ s could be determined. Grazing incidence XRD measurements showed a TiN content of about $70 \%$ for a depth of about $1 \mu \mathrm{m}$. In addition, a lattice constant of $4.224 \mathrm{AA}$ could be measured for the corresponding sample. The macroscopic stress in these directly synthesized coatings is about $-1 \mathrm{GPa}$. In order to avoid cracks, it is necessary to minimize this stress. Further XRD studies have to be carried out in order to quantify all the dependencies.

The treatment with pulsed Nd:YAG laser radiation allows to carry out a fast nitriding process of titanium in a reactive atmosphere. The limiting factors for the coatings are the melting depth in addition to the laser energy and the overlap parameter. In order to create coatings with optimal properties, an overlap of $\Sigma=30$ has to be chosen. For this parameter, the layers formed with sufficient thickness without being brittle and without forming cracks.

Acknowledgments We thank Detlef Purschke for his assistance during the RNRA measurements at the IONAS accelerator. This work was supported by the Deutsche Forschungsgemeinschaft (DFG, grants SCHA 632/4 and SCHA 632/11).

Open Access This article is distributed under the terms of the Creative Commons Attribution Noncommercial License, which permits any noncommercial use, distribution, and reproduction in any medium, provided the original author(s) and source are credited.

\section{References}

1. Bloyce, A, Morton, PH, Bell, T, Surface Engineering of Titanium and Titanium Alloys. ASM International, Materials Park, OH (1994)

2. Man, HC, Cui, ZD, Yue, TM, Cheng, FT, "Cavitation Erosion Behavior of Laser Gas Nitrided Ti and Ti6Al4V Alloy." Mater. Sci. Eng. A, 355 (1-2) 167-173 (2003)
3. Schaaf, P, Landry, F, Lieb, KP, "The Origin of Nitrogen Depth Profiles upon Laser Nitriding of Iron." Appl. Phys. Lett., 74 (1) 153-155 (1999)

4. Carpene, E, Schaaf, P, "Mass Transport Mechanisms During Excimer Laser Nitriding of Aluminum.” Phys. Rev. B, 65 (22) 224111-1-9 (2002)

5. Carpene, E, Flank, AM, Traverse, A, Schaaf, P, "EXAFS Investigation of Laser Nitridation and Laser Carburization of Silicon." J. Phys. D: Appl. Phys., 35 1428-1432 (2002)

6. Carpene, E, Shinn, M, Schaaf, P, "Synthesis of Highly Oriented $\mathrm{TiN}_{x}$ Coatings by Free-Electron Laser Processing of Titanium in Nitrogen Gas." Appl. Phys. A: Mater. Sci. Process., 80 (3) 1707-1710 (2005)

7. Schaaf, P, "Laser Nitriding of Metals." Prog. Mater. Sci., 47 (1) 1-161 (2002)

8. Xin, H, Mridha, S, Baker, TN, "The Effect of Laser Surface Nitriding with a Spinning Laser Beam on the Wear Resistance of Commercial Purity Titanium." J. Mater. Sci., 31 (1) 22-30 (1996)

9. Mori, JC, Serra, P, Martianez, E, Sardin, G, Esteve, J, Morenza, JL, "Surface Treatment of Titanium by Nd:YAG Laser Irradiation in the Presence of Nitrogen." Appl. Phys. A: Mater. Sci. Process., 69 699-702 (1999)

10. György, E, Perez del Pino, A, Serra, P, Morenza, JL, “Depth Profiling Characterisation of the Surface Layer Obtained by Pulsed Nd:YAG Laser Irradiation of Titanium in Nitrogen." Surf. Coat. Technol., 173 (2-3) 265-270 (2003)

11. György, E, Perez del Pino, A, Serra, P, Morenza, JL, "Surface Nitridation of Titanium by Pulsed Nd:YAG Laser Irradiation.' Appl. Surf. Sci., 186 (1-4) 130-134 (2002)

12. Xue, L, Islam, M, Koul, AK, Bibby, M, Wallace, W, "Laser Gas Nitriding of Ti-6Al-4V Part 1: Optimization of the Process." Adv. Perform. Mater., 4 (1) 25-47 (1997)

13. Xue, L, Islam, M, Koul, AK, Bibby, M, Wallace, W, "Laser Gas Nitriding of Ti-6Al-4V Part 2: Characteristics of Nitrided Layers." Adv. Perform. Mater., 4 (4) 389-408 (1997)

14. Landry, F, Schaaf, P, "Simulation and Deconvolution Program WinRNRA for Depth Profiling of Light Elements via Nuclear Resonance Reactions." Nucl. Instrum. Methods Phys. Res. B, 179 (2) 262-266 (2001)

15. Uhrmacher, M, Hofsäss, H, "Ion Accelerator Facilities at the University of Göttingen." Nucl. Instrum. Methods Phys. Res. B, 240 (1-2) 48-54 (2005)

16. Joint Committee for Powder Diffraction Studies (JCPDS)ICDD-PDF2 Database 1997 PCPDFWIN 1.30

17. Prevey, PS, Metals Handbook. ASM International, Metals Park, OH (1986)

18. Lee, T, Ohmori, K, Shin, CS, Cahill, DG, Petrov, I, Greene, JE, "Elastic Constants of Single-Crystal $\mathrm{TiN}_{x}(001)(0.67<=x$ $<=1.0)$ Determined as a Function of $\mathrm{x}$ by Picosecond Ultrasonic Measurements." Phys. Rev. B, 71 (14) 144106-6 (2005)

19. Lichinchi, M, Lenardi, C, Haupt, J, Vitali, R, "Simulation of Berkovich Nanoindentation Experiments on Thin Films Using Finite Element Method." Thin Solid Films, 312 (1-2) 240-248 (1998)

20. Vegard, L, Z. Phys., 517 (1921)

21. Nagakura, S, Kusunoki, T, Kakimoto, F, Hirotsu, Y, "Lattice Parameter of the Non-Stoichiometric Compound $\mathrm{TiN}_{x}$." J. Appl. Crystallogr., 8 (1) 65-66 (1975)

22. Höche, D, Schikora, H, Zutz, H, Queitsch, R, Emmel, A, Schaaf, P, "Microstructure of TiN Coatings Synthesized by Direct Pulsed Nd:YAG Laser Nitriding of Titanium: Development of Grain Size, Microstrain, and Grain Orientation." Appl. Phys. A: Mater. Sci. Process., dx.doi.org/10.1007/ s00339-008-4403-8 in print 
23. Shuja, SZ, Yilbas, BS, Budair, MO, "Modeling of Laser Heating of Solid Substance Including Assisting Gas Impingement." Numer. Heat Transf. A: Appl., 33 (3) 315-339 (1998)

24. Schaaf, P, Han, M, Lieb, KP, Carpene, E, "Laser Nitriding of Iron with Laser Pulses from Femtosecond to Nanosecond Pulse Duration." Appl. Phys. Lett., 80 1091-1093 (2002)
25. Jönsson, B, Hogmark, S, "Hardness Measurements of Thin Films." Thin Solid Films, 114 (3) 257-269 (1984)

26. Burnett, PJ, Rickerby, DS, "The Mechanical Properties of Wear-Resistant Coatings II: Experimental Studies and Interpretation of Hardness." Thin Solid Films, 148 (1) 5165 (1987) 